\title{
Performance Analysis of Inter-Satellite Optical Wireless Communication
}

\author{
Vinod Kiran K, Sarath V S, Vikram Kumar, Ashok K Turuk, Santos K Das* \\ Department of Electronics and Communication Engineering National Institute of Technology Rourkela, India \\ E-mail: *dassk@nitrkl.ac.in
}

\begin{abstract}
The evolution of optical communications systems from long fibers to robust wireless system has led to the idea of connecting two satellites using an optical wireless link. The rapid developments in satellite communication point to the possibilities of satellite networks as a method for them to communicate with each other. The optical wireless communication (OWC) enables the information exchange between satellites at higher speed and also a satellite can act as a relay between two satellites or between a satellite and ground base station. Light signal of high frequency is used as a carrier for the optical link between satellites and lasers are commonly used as a source of light. One of the primary reasons for preferring OWC over RF link is high data rate with acceptable bit error rate (BER). The communication between two lower earth orbit (LEO) satellites is studied under this research work. The quality factor (Q-factor) or BER is used as a key metric to analyze the performance of the inter-satellite link (ISL). This work presents the performance enhancement of the system by using multiple antennas and advanced modulation techniques.
\end{abstract}

Index Terms - Optical Wireless Communication (OWC), Lower Earth Orbit (LEO) Satellites, Photodiode, Quality Factor (Q-Factor).

\section{INTRODUCTION}

The optical wireless link between two satellites or between a satellite and ground station can support high data rate with minimum transmission delay since highfrequency light signal (typically $193.1 \mathrm{THz}$ or wavelength of $1550 \mathrm{~nm}$ ) is used as the carrier. The outer space is assumed to be a vacuum free from the atmosphere hence effect of attenuation is minimal. Optical links can overcome many of the limitations associated with the conventional RF link. The size of the antenna depends on the frequency of the carrier used. It is evident that the transmitting and receiving antenna for the RF system has to be meters wide. An optical communication system requires an antenna of a few centimetres in sizes. Smaller antenna size means small size payload which ultimately results in reduced mass and cost of the satellite, this is the greatest advantage of the optical link [1]. Laser beam width is narrow because of the smaller wavelength of the light signal used [2]. Therefore, signal power loss in case of OWC system is less compared to RF system. The regulations and license to the frequencies that can be used for satellite communication using RF link are not applicable in the case of the optical system [3]. Optical wireless links provide a promising alternative, and a high data rate can be transmitted employing wavelength division multiplexing (WDM) [4]. The major limitation of inter-satellite optical wireless communication (IsOWC) system is that it requires a highly accurate tracking system to make sure that the communicating satellites are aligned with a strict line of sight (LOS).

\section{RELATED WORK}

The number of satellites deployed to space increases year by year. Satellite communication plays a vital role in many areas of applications that are beneficial to human kind. Many of the researchers are working widely to provide a better technology for next generation data requirements. V W S Chan [2] studied that the optical space communication between satellites occurs at very high data rates (greater than $10 \mathrm{Gbps}$ ) is feasible. Sodnik $\mathrm{Z}$ et al. [5] observed that technical advancement in intersatellite optical wireless communication is promising; still it lags when comes to large scale commercial deployment as the operators are skeptical about the efficiency and reliability. The successful deployment of EDRS (European Data Relay Satellite) system and SILEX (Semiconductor laser Inter-Satellite Link Experiment) program made the commercial satellite market to think otherwise and resulted in the use of optical wireless link in space technology for high speed voice and data communication [6]. Noor N H M et al. [7] proposed a multiple transceivers system to provide high bit rate with acceptable BER. Patnaik B et al. [8] proved to achieve a data rate of $400 \mathrm{Gbps}$ over $4767 \mathrm{~km}$ distance for an inter-satellite optical link using coherent optical QPSK technique. Patnaik B et al. [9] designed a 32 channel DWDM system using optical QPSK for free space optical link to achieve a data rate of $40 \mathrm{Gbps}$. Rani et al. [10] have studied the inter satellite vibrations and corresponding link misalignment, pointing errors with corresponding BER was studied. Kaur et al. [11] has evaluated the IsOWC performance with different wavelength under pointing errors.

The motivation behind the research is that the optical wireless link between LEO satellites can form a powerful satellite network of high data rates. This ensures a global coverage in terms of both voice and data communication 
at any place and at any time. Following to introduction at Section I; Section II represents the related work in optical wireless communication in satellite; Section III explains the concept of system modeling, Section IV discusses the simulation results obtained, and Section $\mathrm{V}$ presents the conclusions.

\section{SYSTEM MODELING}

For analyzing the system, various parameters affecting system performance have to be considered. The system parameters such as the distance between the satellites, information bit rate, input power, and wavelength are few variables considered here to optimize the system for satisfactory performance. Q-factor or BER is used as a performance metric to analyze the system. BER is the ratio of the number of bit errors detected in the receiver to the number of bits transmitted. Noise affected signal leads to incorrect decisions being made at the receiver system and hence results in bit errors. BER holds an inverse relation with Q-factor which can be represented as,

$$
B E R=\frac{1}{2} \operatorname{erfc}\left(\frac{Q}{\sqrt{2}}\right) \approx \frac{1}{\sqrt{2 \pi Q}} \exp \left(\frac{-Q^{2}}{2}\right)
$$

where, Q is the Quality factor.

BER is usually too small to quantify in the case of optical domain thus Q-factor is mostly used as a quantifying parameter for the analysis. IsOWC system can be modeled using OptiSystem software. Fig.1 shows a simple IsOWC system which consist three blocks namely transmitter, OWC channel, and receiver. A satellite accommodates both transmitter and receiver block to support duplex communication. The OWC channel corresponds to the environment surrounding of the satellites i.e. outer space. The OWC channel modeled in the software is specifically designed to suit the intersatellite environment [12].

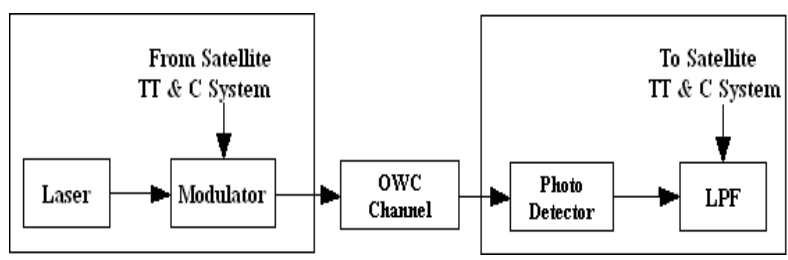

Fig.1. IsOWC System

\section{A. IsOWC Transmitter}

A basic transmitter consists of a pseudo-random bit sequence (PRBS) generator corresponds to a source of information in binary form. This information usually comes from the satellite's telemetry, tracking, and control (TT and C) system. Non-return to zero (NRZ) pulse generator encodes the binary information into the corresponding electrical signal. CW laser is the source of the light carrier produces a continuous unmodulated wave. LEDs can also act as the light source, but lasers are preferred due to its capacity to transmit over longer distances. The power of the light carrier is fixed to $15 \mathrm{dBm}$ and the frequency used is $193.1 \mathrm{THz}$ (corresponding to a wavelength of $1550 \mathrm{~nm}$ ). Mach-Zehnder modulator is a major component of the transmitter system. It is an optical modulator whose function is to vary the intensity of the light from the laser according to the output of the NRZ pulse generator [12].

\section{B. OWC Channel}

The satellites orbit around the earth in outer space and this environment surrounding satellites is the transmission medium. This environment is substituted by the OWC channel component modeled in the OptiSystem tool. The optical antennas at both transmitter and receiver end are part of the OWC channel component. The antenna parameters like antenna gain, optical efficiency, and pointing error factor, etc. can be varied according to the requirements. The optical efficiency of antennas is assumed to be 1 for ideal case. Since the effect of vibration and other factors leading to pointing errors are not studied under this research, the pointing error factor is assumed to be null considering a perfect alignment. The transmitter and receiver antenna gain is assumed to be 0 $\mathrm{dB}$ since satellites are at very higher altitudes above the Earth's atmosphere, there is no attenuation due to atmospheric effects.

\section{IsOWC Receiver}

The receiver section consists of an avalanche photodiode (APD) and a low pass filter (LPF). The photodiode is an optical detector where incident photons excite the electrons inside the diode, resulting in current flow through the output circuit. So, the signal obtained is in electrical form at the output for the corresponding optical input signal. Unlike a photodiode, APD has got internal gain due to avalanche phenomenon. The multiplication factor is set to 3 for the APD used here. Hence, there is no need of an external amplifier at the receiver. APD is perfect to be employed in the optical detection system since for long distance transmission the intensity of the light transmitted decreases towards the receiver end. The dark current is fixed at a default value of $10 \mathrm{nA}$. The frequency of the photodiode is set to 193.1 $\mathrm{THz}$. The function of the LPF is to filter out the unwanted higher frequency signals. Bessel LPF is used with a cutoff frequency 0.75 times the bit rate of the signal. The order of the Bessel filter is set to 4 , and the maximum attenuation of the filter is fixed at $100 \mathrm{~dB}$ [12].

\section{IsOWC System Using Multiple Antennas}

It is observed that high transmitter power and bigger antenna size are needed for communication at high data rates over long distances. So, multiple antennas are used to enhance the system performance. An IsOWC system with four antennas at both transmitter and receiver end is modeled using the OptiSystem software. The results obtained from the system model with multiple transceiver antennas are compared with that of the simple system design. It can be concluded that the introduction of $4 \times 4$ antenna system makes the communication possible up to 
$6000 \mathrm{~km}$ at the data rate of $10 \mathrm{Gbps}$ with acceptable BER [7]. But the accommodation of multiple antennas demands increased the size of the payload. So increasing the number of antennas beyond a limit is not recommended.

\section{E. Modulation Techniques}

The modulation technique used plays a vital role in any form of the communication system whether it is electrical or optical. Therefore, the selection of proper modulation is most important in the case of IsOWC system design. The simplest and commonly used modulation scheme is intensity modulation also referred as direct detection technique (IM/DD), similar to the amplitude modulation in an electrical communication system. IM/DD is less immune to channel noise as the non-predictive channel produces random fluctuation on the optical intensity level. It demands a complex and adaptive thresholding block at the receiver system to reconstruct the original signal with minimum BER. The difficulty in implementation of this complex system leads to the consideration of a simple and better solution. It is better to carry information in the phase or frequency of the carrier signal since the optical intensity level is affected by the noise. The phase shift keying (PSK) based modulation requires no adaptive thresholding scheme, thus offers superior performance compared to the IM/DD system in the presence of channel noise.

\section{F. IsOWC System Using QPSK Modulation}

A single channel IsOWC system using coherent optical QPSK modulation technique is proposed for a bit rate of 40 Gbps. The performance of the system is also compared with the conventional IM/DD techniques [9]. An optical QPSK transmitter is shown in Fig.2. The binary signal from the PRBS generator is interfaced to the PSK sequence encoder which generates the in-phase and quadrature phase signal components. QPSK modulation system consists of an $\mathrm{M}$-ary pulse generator with a value of $\mathrm{M}=4$, and the number of bits per symbol as two. A Mach-Zehnder modulator is used to modulate the highfrequency optical signal using the M-ary pulse generator output signal. The optical carrier signal from the $\mathrm{CW}$ laser is transmitted to the modulator using a fiber coupler. Finally, the output from both the modulators is combined using an optical power combiner to give the optical QPSK modulated signal.

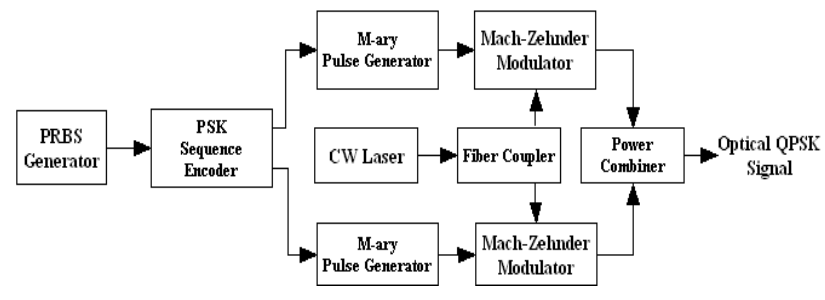

Fig.2. Optical QPSK Transmitter

Fig.3 shows a coherent optical QPSK receiver. The optical QPSK detector comprises of photodiodes and local oscillator (laser source). It coherently detects the transmitted light and converts it into the desired electrical form for further processing. The optical detector is followed by a signal conditioning which consists of three blocks, an electrical amplifier to enhance the signal, an LPF to filter out higher order frequency components, and a decision device to reproduce the original signal from the received signal. The conditioned signal is decoded into a binary form using a PSK decoder.

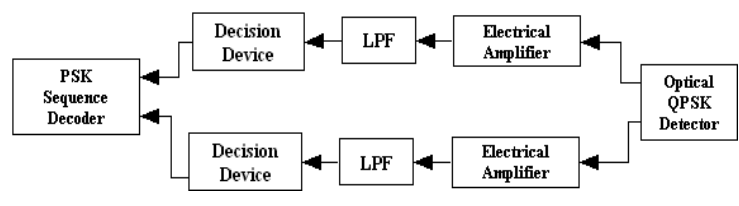

Fig.3. Optical QPSK Receiver

\section{SimULATION RESUlTS AND DisCUSSION}

The objective of this research work covers the analysis of an optical wireless link between satellites, specifically between two LEO satellites. There are few assumptions made to fit this work into a simulation environment. As we know, the environment surrounding the satellites is the outer space which is the propagation medium for the transmitted signal, and it is free from the atmosphere. Thus the channel is free from atmospheric attenuation effects. The effect of particles obstructing the light path is out of the scope. But the presence of small and large particles in the form of meteorites, space dust, satellite debris, etc. in the signal path may lead to scattering effects and thereby results in signal degradation. Attenuation due to Rayleigh and Mie scattering is inversely proportional to the wavelength. Thus, the effect of scattering can be reduced by using longer wavelength. The longest possible wavelength that can be used to mitigate the effect of scattering is $1550 \mathrm{~nm}$. Also, this wavelength of $1550 \mathrm{~nm}$ is compatible with current technology and devices [13]. Proper alignment of satellites to have an LOS path is assumed to study the link performance. Hence, the link performance depends on the various results obtained using the simulation tool, and the channel characteristics are set as per the variable parameters available with the OWC channel component.

An optical link connecting two satellites is modeled using OptiSystem and executed for analyzing the system performance under variable conditions. The parameters like the distance between satellites (range), bit rate and antenna diameter are varied. The maximum range is limited to $6000 \mathrm{~km}$ since the inter-satellite link between LEO satellites is only under consideration. The results obtained by varying different system parameters are analyzed. A satisfactory system performance at $6000 \mathrm{~km}$ range guarantees the same at smaller distances. Therefore, all the discussions are focused on the performance at larger range. The enhanced performance using multiple antenna systems and using QPSK modulation technique respectively is also discussed here.

Three different systems are modeled namely, i) single transceiver system using OOK, ii) multiple transceiver 
system using OOK, and iii) single transceiver system using QPSK. The performance in terms of Q-factor or BER is compared across all these systems.

\section{A. System Performance with Varying Distance between Satellites, Data Rate, and Antenna Diameter}

The bit rate, distance between the satellites and antenna diameter are varied to study the Q-factor obtained in each case. The range of operation is set from $1000 \mathrm{~km}$ up to $6000 \mathrm{~km}$. A very important parameter affecting the system performance is the antenna size which is varied from $15 \mathrm{~cm}$ to $60 \mathrm{~cm}$. The frequency of light carrier used is $193.1 \mathrm{THz}$ (wavelength of $1550 \mathrm{~nm}$ ), and the input signal power is $15 \mathrm{dBm}$. The system is analyzed against four different data rates starting from a value of $10 \mathrm{Mbps}$ to $10 \mathrm{Gbps}$. The modulation scheme employed is a simple on-off keying (OOK) where the intensity of the light carrier is modulated with the information signal to be transmitted. Initially, the system is modeled for a data rate of $1 \mathrm{Gbps}$ and the corresponding Q-factor with different range is plotted for four different values of the antenna dimensions is as shown in Fig.4.

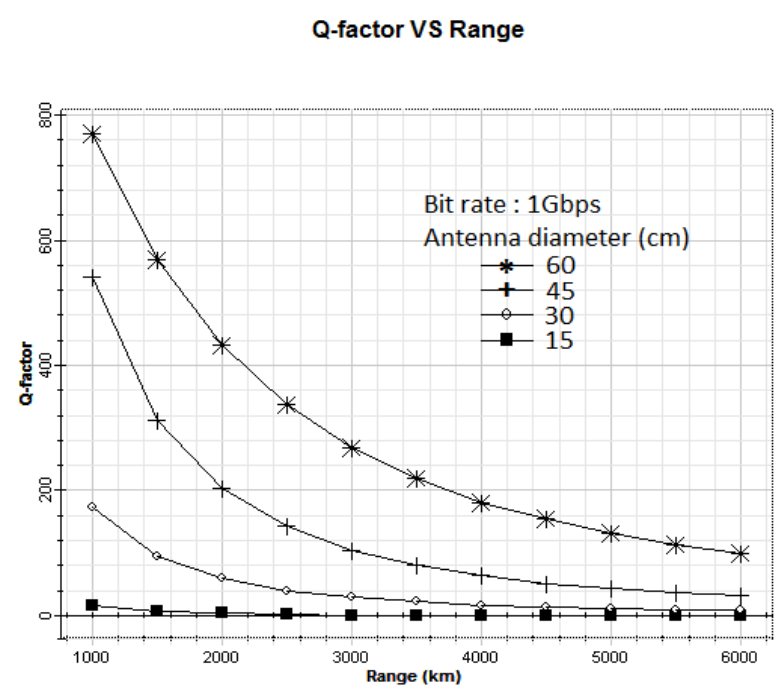

Fig.4. Q-Factor Plotted Against Range for Different Values of Antenna Diameter

It can be observed that at a $6000 \mathrm{~km}$ range with $15 \mathrm{~cm}$ antenna dimension gives a zero Q-factor, whereas a $30 \mathrm{~cm}$ antenna gives the Q-factor of 7.87 and this increase as the antenna size increases. Also, the Q-factor varies inversely with the distance between satellites. It implies that the signal quality degrades as it travels a longer distance. Qfactor holds an inverse relationship with the increase in data rate as shown in Fig. 5. It can be observed that as the bit rate increased the corresponding Q-factor has bee decreased. The smaller antenna of $30 \mathrm{~cm}$ diameter can cover the $6000 \mathrm{~km}$ range with a maximum bit rate of 10 Mbps. The important observation from the results is that a $30 \mathrm{~cm}$ antenna system with a high bit rate of $10 \mathrm{Gbps}$ can cover only a distance of less than $500 \mathrm{~km}$. It can be observed that Q-factor increases with increasing antenna diameter.

\section{Q-factor VS Antenna diameter}

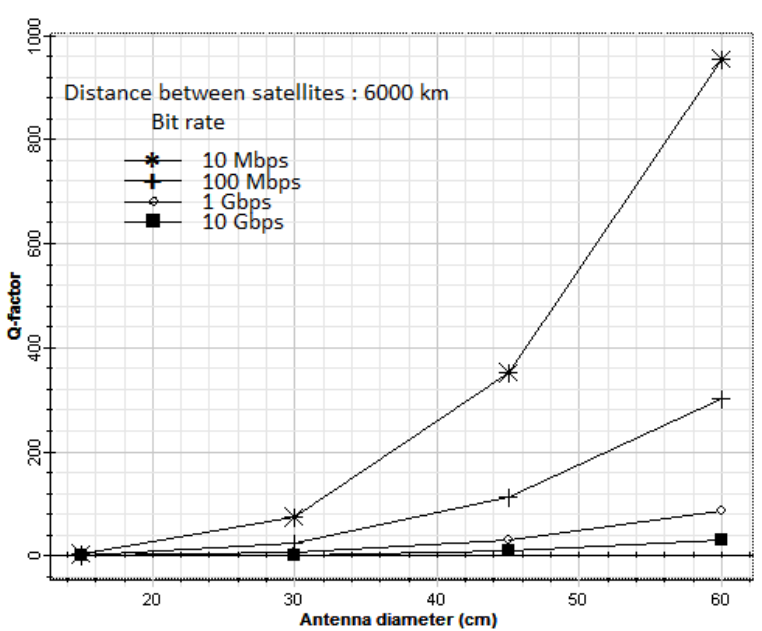

Fig.5. Q-Factor Plotted Against Antenna Diameter for Different Values of Bit Rate

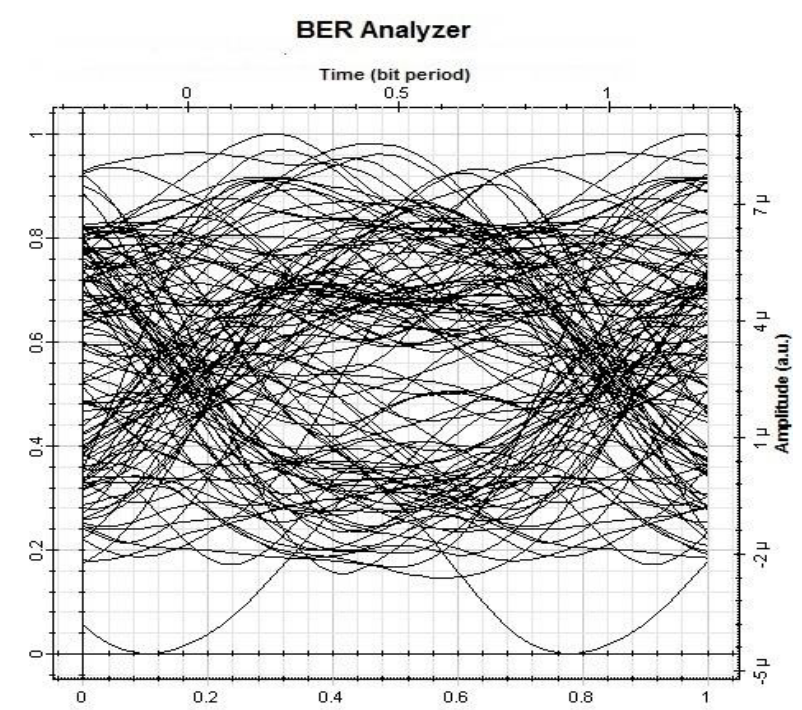

Fig.6. Eye Diagram of a Single Antenna System with a Link Distance of $6000 \mathrm{Km}$

However, antenna diameter cannot be increased beyond a limit since the major advantage of preferring optical link over RF link is the antenna dimension. An alternative solution to cover the longer distance with high data rate is by employing multiple antenna systems or the use of advanced modulation schemes.

\section{B. IsOWC System Performance under Multiple Antennas}

A $4 \times 4$ transceiver system is modeled where each antenna is having a diameter of $30 \mathrm{~cm}$ with an input power of $15 \mathrm{dBm}$. The data rate used is $10 \mathrm{Gbps}$. The eye patterns obtained in the case of a single antenna system and a $4 \times 4$ antenna system are shown in Fig.6 and Fig. 7 respectively. The eye height or opening of the eye represents an insight of the signal quality. Fig.6 shows almost closed eye pattern due to noise affected signal. Fig.7 represents the eye diagram of multiple antenna systems having a sufficient eye height which indicates an undistorted signal. Fig.8 represents the comparison of Q- 
factor for different transmission ranges with the single antenna system and proposed multiple antenna systems. Consider for a $6000 \mathrm{~km}$ distance, $4 \times 4$ antenna system gives a Q-factor of 27.17 compared to the zero Q-factor of a single antenna system. The Q-factor obtained for the multiple antenna systems provides better performance than the single antenna system. This achieves the data transmission of $10 \mathrm{Gbps}$ can be easily realizable using a $4 \times 4$ antenna system.

\section{IsOWC System Performance Using QPSK Modulation}

A system with QPSK modulation scheme is modeled with the antenna diameter $30 \mathrm{~cm}$ and optical signal power of $15 \mathrm{dBm}$ for a data rate of $40 \mathrm{Gbps}$. Fig. 9 shows the original electrical signal to be transmitted from the QPSK modulator. Fig.10 represents the signal recovered at the receiver using a coherent optical QPSK demodulator. The reconstructed signal at the receiver is almost similar to the transmitted electrical signal, which implies that the effect of noise on QPSK modulated signal is much lesser compared to that of simple IM/DD technique. QPSK scheme has zero BER throughout the range under consideration, but BER is increased to the maximum beyond a range for OOK modulation. The data transmission with an acceptable BER is possible with a bit rate of 40Gbps using QPSK modulation as shown in Fig.11. Hence, coherent optical QPSK modulation supports a high data rate transmission for inter-satellite optical wireless communication as compared to that of IM/DD technique. This indicates the tolerance to channel noise is more for the QPSK modulation as the phase of the carrier contains the information to be transmitted. The Q-factor observed is very high in case of QPSK scheme as compared to that of simple intensity modulation. Here, BER is used to compare the performance of both the systems. This can be observed from BER versus range plot for both OOK and QPSK modulation technique from Fig.11.

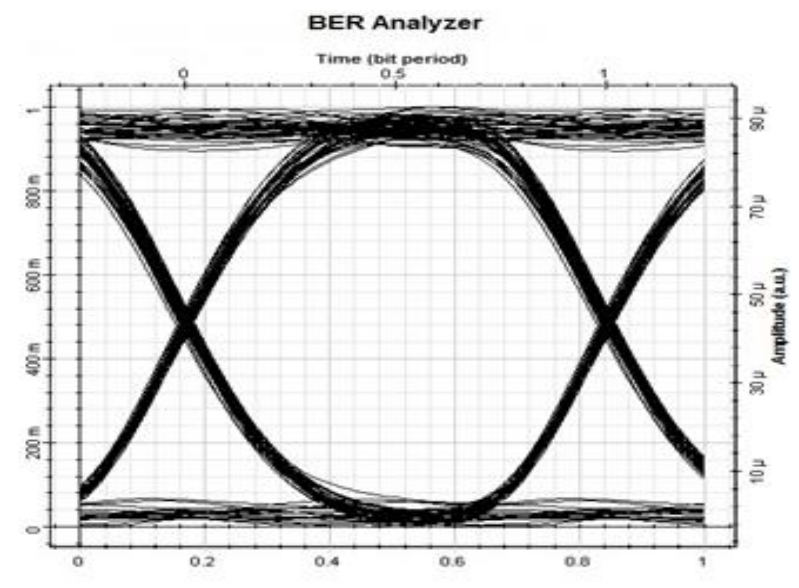

Fig.7. Eye Diagram of A $4 \times 4$ Antenna System with a Link Distance of $6000 \mathrm{Km}$
Q-factor VS Range

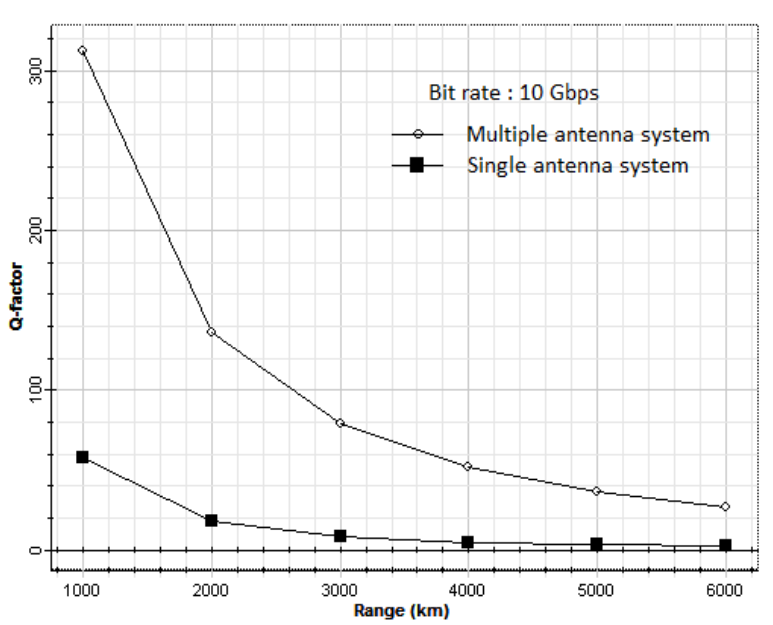

Fig.8. Performance Comparison between $4 \times 4$ Antenna Systems and Single Antenna System

Oscilloscope Visualizer_1

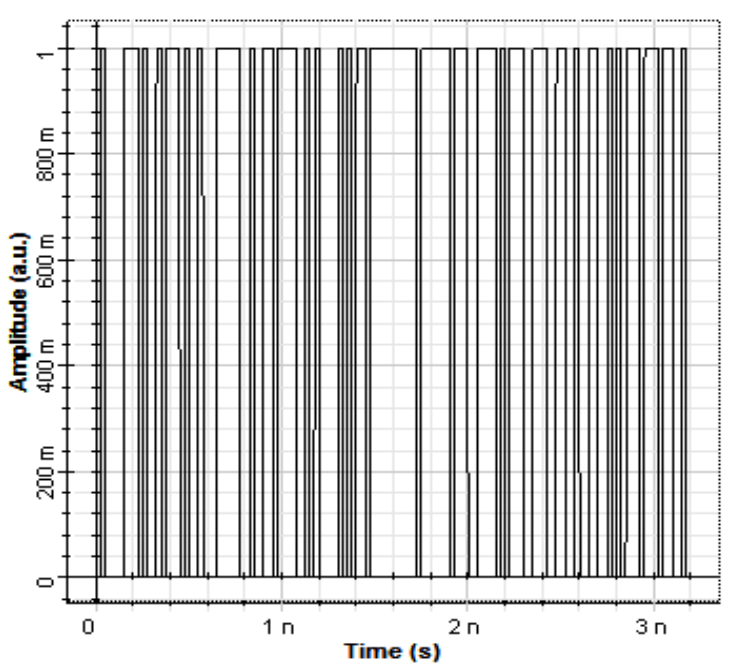

Fig.9. Signal Fed to the Transmitter

Oscilloscope Visualizer_2

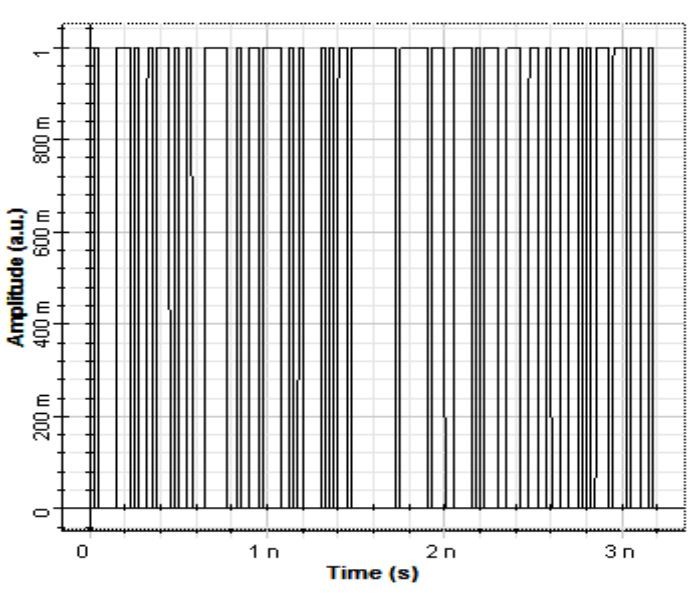

Fig.10. Signal Reconstructed at the Receiver 
BER VS Range

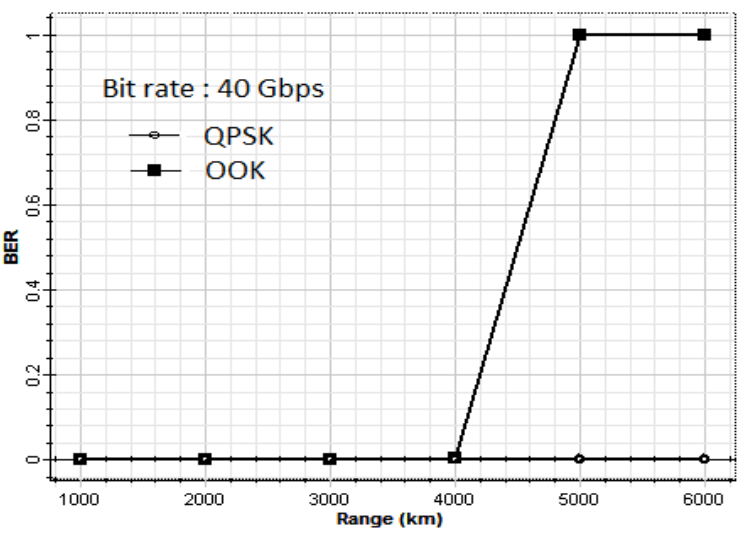

Fig.11. Performance Comparison between QPSK and OOK Technique Used

\section{CONCLUSION}

This work analyzes the performance of the optical link between satellites by varying the parameters affecting the system performance. Inter-satellite communication using optical link can support a higher speed and much longer distance compared to RF links. The proposed system can support a data rate of 10 Gbps by employing a $4 \times 4$ transceiver system over $6000 \mathrm{~km}$. Also, a higher data rate of $40 \mathrm{Gbps}$ is achieved using the QPSK modulation technique in an IsOWC system.

\section{ACKNOWLEDGMENT}

This research work is supported by Department of Electronics and IT (Deity), Ministry of Communications and IT, Government of India under Visvesvaraya $\mathrm{PhD}$ Scheme for Electronics and IT (Grant no: PhDMLA/4(13)/2015-16).

\section{REFERENCES}

[1] Z. Sun, Satellite Networking - Principles and Protocols, UK: John Wiley \& Sons, 2005.

[2] V. W. S. Chan, "Optical Satellite Networks," Journal of Lightwave Technology, Vol. 21, No. 11, pp. 2811-2827, 2003.

[3] Kaushal, Hemani, and Georges Kaddoum. "Optical communication in space: Challenges and mitigation techniques," Communications Surveys \& Tutorials, IEEE, 2016.

[4] Lutz, Erich, Hermann Bischl, Harald Ernst, Florian David, Matthias Holzbock, Axel Jahn, and Markus Werner, "Development and future applications of satellite communications," Emerging Location Aware Broadband Wireless Ad Hoc Networks, Springer, pp. 231-246, 2005.

[5] Z. Sodnik, B. Furc and H. Lutz, "Optical inter-satellite communication," J. Sel. Top. Quantum Electron, IEEE, Vol. 16, No. 5, pp. 1051-1057, 2010.

[6] Z. Sodnik, B. Furch and H. Lutz, "Free-space laser communication activities in Europe: SILEX and beyond," Lasers and Electro-Optics Society, IEEE, pp. 78-79, 2006.

[7] N. H. M. Noor, A. W. Naji and W. Al-Khateeb,
"Theoretical analysis of multiple transmitters and receivers on the performance of free-space optics (FSO) Link," International Conference on Space Science and Communication (IconSpace), IEEE, pp. 291-295, 2011.

[8] B. Patnaik and P. K. Sahu, "Inter-satellite optical wireless communication system design and simulation," IET Communications, Vol. 6, No. 16, pp. 2561-2567, 2012.

[9] B. Patnaik and P. K. Sahu, "Novel QPSK Modulation for DWDM Free Space Optical Communication System," Wireless Advanced (WiAd), IEEE, pp. 170-175, 2012.

[10] Rani, Michael, and Shanthi Prince. "A study on intersatellite optical wireless communication and its performance analysis," International Conference on Devices, Circuits and Systems, IEEE, 2012.

[11] Kaur, Navjot, and Gaurav Soni. "Performance analysis of inter-satellite optical wireless communication (IsOWC) system at $980 \mathrm{~nm}$ and $1550 \mathrm{~nm}$ wavelengths," International Conference on Contemporary Computing and Informatics, IEEE, 2014.

[12] A. H. Hashim, F. D. Mahad, S. M. Idrus, A. Sahmah and A. Supa, "Modeling and performance study of intersatellite optical wireless communication system," International Conference on Photonics (ICP), IEEE, pp. $1-4,2010$.

[13] E. Leitgeb, T. Plank, M. S. Awan, P. Brandl, W. Popoola, Z. Ghassemlooy, F. Ozek and M Wittig, "Analysis and evaluation of optimum wavelengths for free-space optical transceivers," International Conference on Transparent Optical Networks (ICTON), IEEE, 2010.

\section{Authors' Profiles}

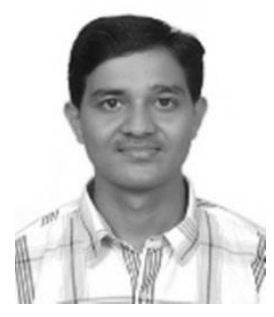

Vinod Kiran $\mathbf{K}$ is pursuing Ph.D. from National Institute of Technology, Rourkela, India. He received M-Tech in Communication and Networks from National Institute of Technology, Rourkela, India. His research interest includes Optical Networking, Free Space Optical Communication, and Sensor Networking.

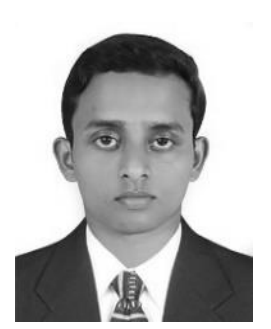

Sarath $\mathbf{V} \mathbf{S}$ is pursuing M-Tech at National Institute of Technology, Rourkela, India. He received B-Tech in Electronics and Communication Engineering from Government College of Engineering, Kannur, India in 2010. His research interest includes Optical Networking, Satellite communication, and Wireless networks.

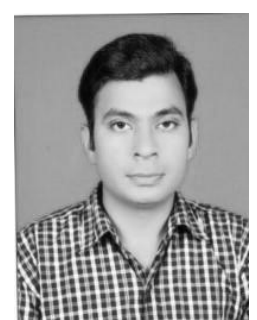

Vikram Kumar is currently pursuing Ph.D. in the field of optical WDM Networks at National Institute of Technology, Rourkela, India. He received M-Tech in the specialization of Communication System engineering from KIIT University, Bhubaneswar, India in 2014. His research interest includes Optical Networking, WDM Networks, and Wireless communication. 


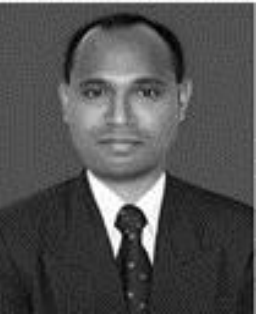

Ashok Kumar Turuk is an Assistant Professor at the Department of Electronics and Communication Engineering, National Institute of Technology, Rourkela, India. He received his $\mathrm{Ph} . \mathrm{D}$ from IIT Kharagpur, India, in 2005. His research interest includes Optical, Wireless Network, Network Security, Mobile Computing, and Embedded System.

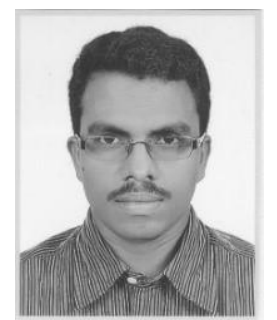

Santos K Das is an Assistant Professor at the Department of Electronics and Communication Engineering, National Institute of Technology, Rourkela, India. He received MS from IISc Bangalore, India, in 2002. He has worked in a number of organizations both in India as well as in abroad in various capacities. He has served on the Program Committee of a number of international conferences. He is a member of the IEEE. His research interest includes Computer, Sensor, Optical Networking, and Embedded system.

How to cite this paper: Vinod Kiran K, Sarath V S, Vikram Kumar, Ashok K Turuk, Santos K Das,"Performance Analysis of Inter-Satellite Optical Wireless Communication", International Journal of Computer Network and Information Security(IJCNIS), Vol.9, No.4, pp. 22-28, 2017.DOI: 10.5815/ijcnis.2017.04.03 